\title{
Computer Modeling of a Spinal Reflex Circuit
}

\author{
Bruno L. Dalcin ${ }^{1}$, Frederico Alan Cruz ${ }^{2}$, Célia Martins Cortez ${ }^{2}$, and Emmanuel P. L. Passos ${ }^{3}$ \\ ${ }^{1}$ Depto de Fisiologia, Universidade Federal do Rio de Janeiro, Rua Frei Caneca, 94, 20211-170, Rio de Janeiro, RJ \\ ${ }^{2}$ Depto de Ciências Fisiológicas, Universidade do Estado do Rio de Janeiro, \\ Av. Prof. Manuel de Abreu, 444, 20551-170, Rio de Janeiro, RJ and \\ ${ }^{3}$ Depto de Engenharia Elétrica, Pontifícia Universidade Católica do Rio de Janeiro, R. Marquês de São Vicente, 225, Rio de Janeiro, RJ
}

Received on 14 November, 2004. Revised version received on 13 September, 2005

\begin{abstract}
We used a set of properties of the interactions among the spinal neurons in order to develop a computer model for a spinal reflex circuit. The model equations take into account the synaptic characteristics of the somatodendritic membrane of neurons in a morphofunctional unity of the spinal reflex activity. This model is based on the idea that the responses of spinal alpha-motoneurons to a sensorial stimulation can be modulated by the serial activation of a motor command chain. We developed a Fortran program for simulating a physiological situation. The results are discussed in terms of available experimental data for the motoneuron firing rate.
\end{abstract}

\section{INTRODUCTION}

Mathematical modeling has been widely used in biological and biomedical sciences [1,2]. Computer modeling techniques in neuroscience have been used to study neurophysiologic circuits [3,4], leading to the investigation of mechanisms involved with the control of the locomotion system. Several models have been proposed to explain these mechanisms [5], and each one of them has contributed in different ways to the understanding of several aspects of the basic biology and function of the nervous system [6].

The modeling of neurophysiological circuits may involve the application of artificial neural network techniques [7]. However, the computer modeling of these circuits frequently requires information on certain physiological or anatomical characteristics of the nervous system which are not accessible to direct measurements [Prentice et al. 2001]. Therefore, works of computer simulation are often based on subjective data from the literature [9]. In fact, more precise values of some characteristic parameters of biomembranes and neuronal interactions are not easily available in the literature [10], and we may be forced to use theoretical model estimates for these values $[11,12,13]$. In spite of the problems related to data deficiency, system engineering has contributed to establishing theoretical models and to applying functional concepts of the nervous system at several levels of physiologic complexity $[13,14]$. Important applications are related to the development of motor and sensorial neuroprothesis, including the simulation of biological circuits with increasing degrees of complexity and automation $[6,15]$. However, additional and more detailed studies are still required to overcome the deficiency of quantitative data related to neuronal interactions.

In this paper, we present a detailed description of a model for the spinal reflex circuit, taking into account the synaptic characteristics of the somatodendritic membrane of neurons in a morphofunctional unity of spinal reflex activity. We develop a Fortran 77 based program in order to simulate a physiological situation. The results are discussed on the basis of experimental data for the motoneuron firing rate.

\section{BASIC MORPHOPHYSIOLOLOGY OF THE SPINAL REFLEX}

There are two general kinds of tissues in the central nervous system $(C N S)$, gray matter and white matter. In the gray matter there are nerve cell bodies, dendrites covered with synapses, and axons. Neurons in gray matter organize either as surface layers named cortex or as inner neuron clusters, which are named nuclei. The white matter is basically formed by bundles of axons (or nervous fibers). Its whitish look is due the sheath of myelin involving the axons. The spinal cord gray matter is the integrative area for the spinal reflexes and other motor functions. Sensory signals enter the spinal cord through the sensory nerve roots. They have two different destinations, (a) the gray matter of the spinal cord, which is the terminal of some sensory fibers or their collaterals, and (b) the higher levels of the nervous system, reaching the supra-segmental areas. The brain is considered to be the supra-segmental nervous system, while the spinal cord and the brain stem form the segmental nervous system $[16,17]$.

In the spinal cord gray matter there are sensory neurons, anterior motor neurons $(A M N)$ and interneurons (IN). $A M N$ sgre located in the ventral horns of the cord gray matter, and their axons leave the cord via ventral roots and innervate skeletal muscles. There are two types of AMNs, the alpha motor neurons $(\alpha M N)$ and the gamma motor neurons $(\gamma M N)$. One $\alpha M N$ single nervous fiber innervates from three to several hundred skeletal muscle fibers. One nervous fiber and its muscle fibers are collectively called the motor unit. Individual muscles are composed of numerousmotor units. Fibers of $\gamma M N$ innervate special skeletal muscle fibers called intrafusal fibers, which are part of the sensory receptor called muscle spindle $[16,18]$.

The INs are very small, more numerous than $\alpha \mathrm{MN} s$ and highly excitable. The INs are widely interconnected among them, and many of them directly innervate the $A M N$. Interconnections among $I N \mathrm{~s}$ and $A M N \mathrm{~s}$ are responsible for many of the integrative functions of the spinal cord. Most of the signals from the spinal nerves or signals from the brain are transmitted first through INs, and then reach the AMNs $[19,20]$.

In the cord white matter there are several longitudinal tracts formed by ascending and descending nerve fibers. The corticospinal tract is an important descending motor way, which 
originates in the brain cortex and terminates in the gray matter of a cord segment. The other descending tracts involved with motor control originate from several brain stem areas.

A basic reflex circuit starts from a type Ia nerve fiber originating in a muscle receptor and entering the dorsal root of the spinal cord [21]. Then, one branch of this sensory fiber connects directly with $\alpha M N$, which sends nerve fibers back to the same muscle. It is thus a monosynaptic reflex circuit, which presents almost no delay between spinal input and output signals after excitation of the receptor [22].

Multisynaptic reflex circuits involved in pathways from IN to $\alpha M N$ include Renshaw cells (RSC). They are interneurons located in the ventral horn of the spinal cord, in close association with the $\alpha M N s$ [23]. Collateral branches from the $\alpha M N$ axon are connected to the adjacent RSC. They transmit inhibitory signals to the next $\alpha M N$, and the stimulation of each $\alpha M N$ tends to inhibit the surrounding $\alpha M N$. For a large number of synapses in the multisynaptic pathways, there will be a longer delay between spinal input and output signals, because of the period of time required for completing all of the synaptic events. This synaptic delay is usually of about $0.5 \mathrm{~ms}$ [16].

Spinal reflex responses modulated by hierarchical motor control are attributed to serial activation of a motor command chain [24]. After the connection in the cord gray matter, the sensory fiber collaterals proceed to higher segmental levels and supra-segmental areas.

\section{THE MODEL AND THE METHOD}

A schematic representation of the neural circuit model for the spinal reflex circuit is shown in Fig. 1. As illustrated in this figure, the signals generated in elementSgo directly to element $M N$ through V1, and also proceed to higher levels of the nervous system through V5. Higher levels (supra-segmental areas) of the central nervous system are represented as element $S S$. After signal processing in $S S$, the outputs follow two distinct descending pathways, V2 and V4, which terminate at elements $M N$ and $I N$, respectively. Element $I N$ has an inhibitory action on element $M N$, and modulates its firing rate. After input processing, $M N$ sends outputs to element EF via V6. The circuit elements and their basic equations are described as follows.

Element $S$ represents the sensory receptor. The $S$ output denotes action potentials $(A P)$ generated from transduction of stimuli; they are represented by Dirac delta functions $[25,26]$. The $A P$ frequency ( $S$ output) is related the stimulus intensity, so

$$
S(t)=\sum_{i=1}^{n} \delta\left(t-t_{i}\right)
$$

where $\delta(\mathrm{t})$ is a Dirac delta function and $t_{i}$ denotes the occurrence of the $i$ th $A P$ at the sensory element. The interval between successive $A P$ s is a random Gaussian process (truncated for $t \leq 0$ ), where the mean is equal to the standard deviation.

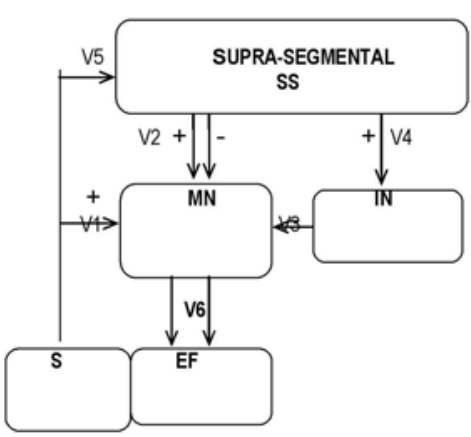

FIG. 1: Schematic representation of the neural circuit as a model representative of a spinal reflex circuit: $S=$ sensorial receptor; $S S=$ supra-segmental area + motor structures of brain stem; V2 and V4 $=S S$ outputs; $I N=$ interneurons pool; $M N=\alpha$-motoneurons; $\mathrm{V} 1=$ $M N$ input from $R ; \mathrm{V} 5=M N$ input from $S S ; \mathrm{V} 3=$ input from $I N$; V5 $=M N$ output; $E F=$ contractile element. The signal $(+)$ refers to excitatory synapses and (-) to inhibitory synapses.

Element SS represents a pool of twenty neurons distributed throughout several areas of the supra-segmental and brain stem, and their axons terminate directly on spinal neurons. Ten of the twenty SS neurons are directly connected to the element $I N$ and the other ten are connected to the element $M N$. Each neuron fiber makes ten excitatory synapses with the element $M N$ or with the element $I N$, i.e., element $S S$ makes one hundred connections with the element $M N$ and one hundred connections with $I N$.

Element IN represents a pool of ten interneurons located in the gray matter of the spinal cord. The interneurons normally receive the excitatory outputs of sensory and motor areas located in the supra-segmental and segmental levels. Each interneuron of the element $I N$ makes ten inhibitory synapses with the element $M N$, and its output signals are able to modulate the firing $M N$ frequency. As already mentioned above, each fiber from element $S S$ makes ten excitatory synapses with element $I N$.

Element $M N$ represents a motoneuron located in the ventral horn of the cord gray matter. The motoneuron and all of its associated muscle fibers collectively form a motor unit. Element $M N$ is able to integrate the signals from elements $\mathrm{S}, I N$ and $S S$. The inputs from elements $S$ and $S S$ produce excitatory effects on $M N$, while inputs from $I N$ generate inhibitory effects.

The general equation for the excitatory effect on a neuron is given by 


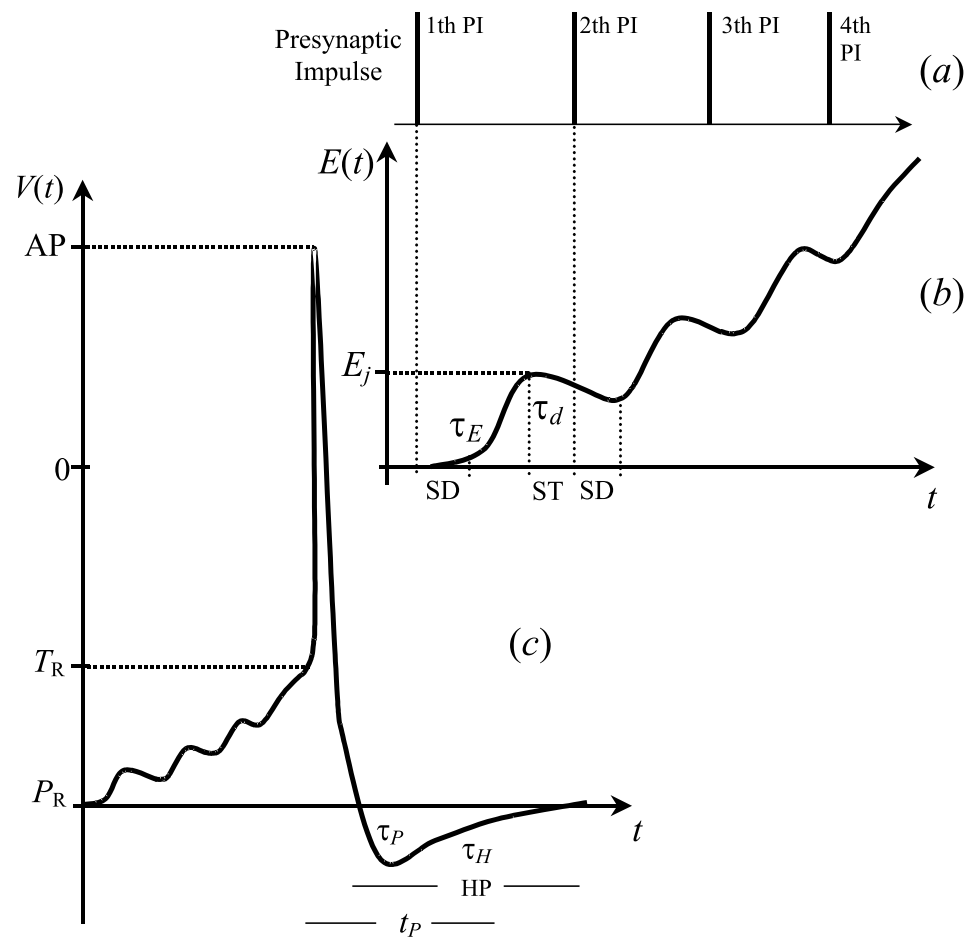

FIG. 2: (a) Schematic representation of a sequence of four presynaptic impulses (PIs) in the $j$ th synapse, (b) the increase of the excitatory synaptic effect, $\mathrm{E}(t)$, after each PI. SD is the synaptic delay between each PI and the beginning of the excitatory postsynaptic potential $\left(\mathrm{E}_{j}\right)$. It increases exponentially along $1 \mathrm{~ms}$, according to the first term of Eq.2, and decays during a "silent time" (ST), according to the second term of the same equation. (c) Schematic representation of the increase of $V(t)$ until the PA firing, according to Eqs. 4 . $P_{R}$ is the rest potential, $\tau_{P}$ is the time constant at the hyperpolarization period (HP) after $A P$ firing, $T_{R}$ is the threshold potential at rest, $\tau_{H}$ is the decay constant at relative refractory period, and $t_{p}$ is the absolute refractory period.

$$
E(t)=\sum_{j}^{n} w_{S E_{j}} E_{j}\left[\sum_{i=1}^{q} \exp \left[-\left(t-t_{i}\right) / \tau_{E}\right]-\sum_{k=1(k \neq i)}^{r} \exp \left[-\left(t-t_{k}\right) / \tau_{d}\right]\right]_{j},
$$

where $E_{j}$ is the characteristic amplitude of the excitatory postsynaptic potential $(E P S P), \mathrm{n}$ is the full amount of synapses, $w_{S E j}$ is the characteristic synaptic weight of the $j$ th synapse, and $t_{i}$ denotes the time of arrival of $i$ th presynaptic $A P$ plus synaptic delay. Note that, after the arrival of the impulse in the terminals of the presynaptic fiber, there is a synaptic delay after which time the postsynaptic potential $(P S P)$ begins to appear; $t_{k}$ denotes the time of absence of a presynaptic im- pulse since the last presynaptic impulse. The number of input synapses is represented by $q$, and $r$ is the total time of absence of presynaptic impulses; $\tau_{E}$ is the time constant and $\tau_{d}$ is the decay constant. Figs. $2 \mathrm{a}$ and $2 \mathrm{~b}$ show a schematic representation of a time series of $\mathrm{E}(\mathrm{t})$ and the generator presynaptic impulses.

The inhibitory effect on element $M N$ is represented by

$$
I(t)=\sum_{j}^{n} w_{S I_{j}} I_{j}\left[\sum_{i=1}^{p} \exp \left[-\left(t-t_{i}\right) / \tau_{I}\right]-\sum_{k=1(k \neq i)}^{s} \exp \left[-\left(t-t_{k}\right) / \tau_{d}\right]\right]_{j},
$$

where $I_{j}$ is the characteristic amplitude of the inhibitory post- 
tic weight of the $j$ th inhibitory synapse. In this equation, $\mathrm{n}$ represents the total number of inhibitory synapses, and $p$ is the total number of synaptic inputs from element $I N$. The total time of absence of presynaptic impulses is denoted bys, and $\tau_{I}$ is a time constant.

Element $\boldsymbol{E F}$ represents the contractile elements responding to signals from $M N$.

As we can see in Eqs. 2 and 3, our model exhibits two characteristic types of qualitative synaptic behavior, temporal and spatial summations. Successive postsynaptic potentials of a rapidly occurring presynaptic are summed, which is is called temporal summation. Spatial summation results from the summation of postsynaptic effects generated by simultaneously discharging presynaptic terminals.

Eq. 2 illustrates the temporal and spatial summations of excitatory effects on a neuron, and Eq. 3 shows temporal and spatial summations of inhibitory effects. In these equations, the first exponential refers to the arrival of the presynaptic impulses, which is related to the time constant of the rising phase and the time-to-peak of the PSP. The second exponential refers to the PSP decay during the intervals between successive presynaptic impulses. We assumed that postsynaptic membranes become highly permeable during $1 \mathrm{~ms}(\tau=1.3)$, and that the produced postsynaptic effects persist for $8 \mathrm{~ms}$, falling exponentially along this time interval (see Fig. 2b).

We consider that an $A P$ in the postsynaptic neuron is a product of a response to a superthreshold stimulation, but a single impulse reaching the presynaptic terminal is assumed to be a subthreshold stimulus. It is known that the neurotransmitter substance released by a single presynaptic terminal is able to generate an excitatory postsynaptic potential no larger than 1 $\mathrm{mV}$, but a potential of 15 to $20 \mathrm{mV}$ is required for the synaptic firing. However, as a terminal fires, the released neurotransmitter substance opens the membrane channels for $1 \mathrm{~ms}$ or so. Since the postsynaptic potential lasts up to $15 \mathrm{~ms}$, a second opening of the same channel can increase the postsynaptic potential to a still larger level $[16,17]$. So, the intrasomal potential of postsynaptic neurons becomes about $1 \mathrm{mV}$ more positive for each added excitatory discharge, the firing threshold is reached, and an $A P$ is generated on the postsynaptic neuron axon.

As simultaneous inhibitory and excitatory postsynaptic potentials produced by widely distributed terminals can summate, $M N$ fires when a sufficiently large depolarizing effect is provided at the input, and the plasmatic membrane potential, $\mathrm{V}(t)$, becomes larger than the threshold potential $\mathrm{T}(t)$. In our model, these potentials are given by

$$
\begin{aligned}
& V(t)=P_{R}+\left(P_{o}-P_{R}\right) \exp \left[-\left(t-t_{p}\right) / \tau_{P}\right]+E(t)+I(t) \\
& T(t)=\mathrm{T}_{o}+\left(\mathrm{T}_{o}-\mathrm{T}_{R}\right) \exp \left[-\left(t-t_{p}\right) / \tau_{H}\right]
\end{aligned}
$$

where $P_{R}$ is a resting potential, $P_{o}$ is the synaptic reversal potential, $\tau_{P}$ is the time constant at the hyperpolarization period after the $A P$ firing, $T_{R}$ is the threshold potential at resting, $T_{o}$ is the threshold after $A P, \tau_{H}$ is the decay constant at relative refractory period, ant $t_{p}$ is the absolute refractory period. In Fig. $2 c$, we give a schematic representation of $V(t)$.
We now consider that $M N$ fires if $\mathrm{V}(\mathrm{t}) \dot{\mathrm{T}}(\mathrm{t})$, the program counts a spike, and a new processing cycle begins, but the dynamics of $\mathrm{V}(\mathrm{t})$ is still described by Eq. $4(a)$.

Equations $4(a)$ and $4(b)$ describe the temporal dynamics of a plasmatic membrane potential of a postsynaptic neuron. Eq. 4 (a) refers to the change in amplitude of the membrane potential resulting from the sum of inhibitory and excitatory effects, taking into account the influence of the absolute refractory period and the time constant after the hyperpolarization period. Eq. $4(b)$ refers to the temporal dynamics of the threshold potential due to each $A P$ fired in a postsynaptic neuron, considering the absolute refractory period and the decay constant at relative refractory period.

Equations (2) to (4) allow the inclusion in this problem of the following synaptic characteristics.

The facilitation mechanism is associated with Eq. 2, since it contains the spatial summation. According to this mechanism, signals from the supra-segmental areas can facilitate the element $M N$, so that they are able to respond quickly and easily to signals arriving from element $S$. In this case, the membrane potential is closer than normally to the firing threshold, but not yet to the firing level. So, a signal entering $M N$ from some other via can then excite it very easily.

As we can note in Eq. 3, the inhibitory effect produced by $I N$ on $M N$ is assumed to be dependent on time. It increases with the activity expansion from the supra-segmental via. This increase reproduces the natural increase during the adaptive process along the uninterrupted activation of the spinal reflex circuit.

The synaptic after-firing mechanism [16] can also be taken into account in this problem, since Eqs. 4 consider the afterfiring changes in the membrane potential and firing threshold, as well as the relative refractory period. This mechanism makes it possible that a single instantaneous input provokes a sustained signal output during some milliseconds, which might result in repetitive firings. If one impulse arrives at the presynaptic terminal in this period of time, its postsynaptic effect can be summed in order to keep the output signal, and the firing threshold can be reached again.

All simulation programs were written in Fortran 77 (Visual Fortran 95 for Windows), compiled and run on PC DOS computers. In those programs, we used numerical values for the parameters found in the literature (Table 1). Some of those values were obtained from experimental studies. The main program accepts input parameters from disk files and writes output data to a disk file. This program was able to estimate postsynaptic effects using Eqs. 2 and 3 at each millisecond, and then using the results in Eqs. 4. Time series of $M N$ membrane potentials resulting from simultaneous activities of the excitatory and inhibitory synapses were estimated during 200 ms.

In addition of the synaptic characteristics described above, neurons of our model are still conceived as presenting some features which are usually found in the synaptic transmission.

When a rapidly repetitive series of impulses stimulates a neuron and then a rest period is allowed, it can be even more responsive than normally to a subsequent stimulation. This is called post-tetanic facilitation. According to the literature, 
Table 1. Some values for neurobiologic parameters

\begin{tabular}{lcc}
\hline Parameter (symbol) & Value & Ref. \\
\hline MN rest potential $\left(P_{R}\right)$ & $-65 \mathrm{mV}$ & {$[16]$} \\
MN reversal potential $\left(P_{o}\right)$ & $+10 \mathrm{mV}$ & {$[35,34]$} \\
Time constant of IPSP $\left(\tau_{I}\right)$ & $1.0 \mathrm{~ms}$ & {$[18]$} \\
Threshold potential at rest $\left(T_{R}\right)$ & $-40 \mathrm{mV}$ & {$[36]$} \\
Threshold potential after firing $\left(T_{o}\right)$ & $-10 \mathrm{mV}$ & {$[36]$} \\
Decay time constant of refractory period $\left(\tau_{H}\right)$ & $1.2 \mathrm{~ms}$ & {$[36]$} \\
IPSP $(I)$ & $-72 \mathrm{mV}$ & {$[34]$} \\
AP time interval & $1.3 \mathrm{~ms}$ & {$[34]$} \\
\hline
\end{tabular}

this facilitation period can last from a few seconds in some neurons to several hours in others [16]. In our program, we implemented the occurrence of the post-tetanic facilitation after $1 \mathrm{~s}$ of a rapidly repetitive series of impulses (frequency higher than $250 \mathrm{~Hz}$ ) on $M N$ and $I N$, assuming a reduction in $\tau_{P}$ of $5 \%$ after each $M N$ firing.

As in all excitatory synapses, in our problem there can be a fatigue of the synaptic transmission if the neuron is repetitively excited at a rapid rate. In this case, the neuron firing number becomes progressively smaller with the increasing of the excitation time. Usually, the fatigue occurs in a few seconds to a few minutes. Our system was programmed to fatigue 3 seconds after repetitive excitation (frequency higher than $250 \mathrm{~Hz}$ ), because the threshold potential $H_{R}$ begins to decrease $0.02 \%$ after each $M N$ firing.

\section{RESULTS AND DISCUSSION}

The model that we are proposing incorporates the excitatory and inhibitory properties of the somatodendritic membrane of neurons in a morphofunctional unity of the spinal reflex activity. This model takes into account a set of known synaptic characteristics, including certain mechanisms of temporal and spatial bioelectric responses, as the synaptic after firing, the adaptive process, the facilitation, the post-tetanic facilitation, and the synaptic fatigue. The physiological properties of digitalling and autopropagability of the $A P$ are represented by Dirac delta functions. Routines to calculate postsynaptic effects for the circuit elements were established from Eqs. 2 and 3, using Eqs. 4a and $4 \mathrm{~b}$ for estimating the time series of the membrane potential. The summation of inhibitory and excitatory effects was performed by a suitable computer program.

Random depolarizing signals from elements $S$ and $S S$ are assumed to reach element $M N$ through 10 and 100 synapses, respectively. ElementSalso sends outputs to element $S S$, but we did not model the processing within this element. However, we assumed that element $S S$ can send a random output to elements $M N$ and $I N$, but each $S S$ output only reaches both elements, $M N$ and $I N, 15 \mathrm{~ms}$ after the $S$ firing. So, the impulses $S S$ to $M N$ are the impulses which $S S$ has received from $\mathrm{S}$, with a delay of $15 \mathrm{~ms}$. This was assumed on the basis of the time interval required for one signal to travel via the $S$ $S S$ spinal pathway to reach $M N$ [16]. The element $M N$ also receives inhibitory signals from $I N$ through 100 synaptic connections.
The output of element $I N$, which is generated by processing random signals $(200 \mathrm{~Hz})$ from $S S$ during $200 \mathrm{~ms}$, can be seen in Fig. 3. The excitatory synaptic weight $\left(w_{S E j}\right)$ on $I N$ is 0.9 , the synaptic delay is $0.6 \mathrm{~ms}$, and the other parameter values are given in Table 1. Fig. 3 shows the result of this processing as a random collection of $A P$ s with a mean frequency of 45 $\mathrm{Hz}$. This result is in overall agreement with the literature. The measured values in interneurons of decerebrate cats vary from 20 to $110 \mathrm{~Hz}$ [26]. According to Cleland and Rymer [27], certain interneurons within the cord gray matter have a variable and elevated spontaneous activity with mean frequency $30.3( \pm 20.1) \mathrm{Hz}$, and sensory-evoked responses larger than $400 \mathrm{~Hz}$.

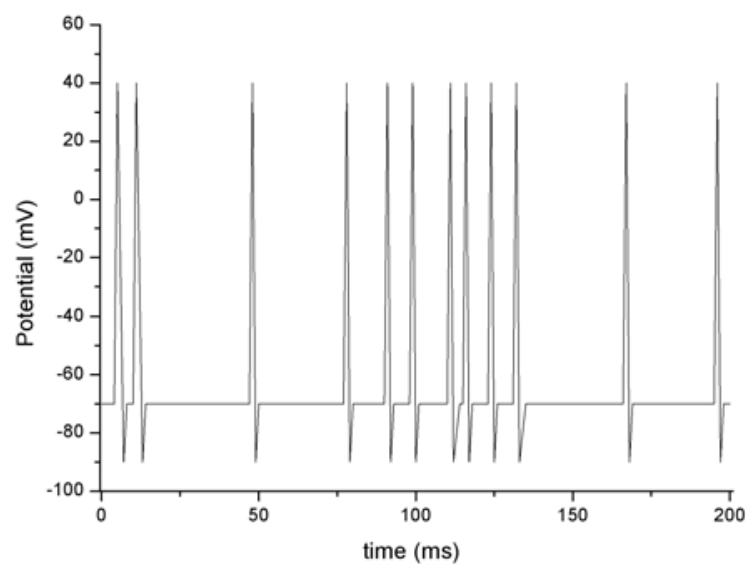

FIG. 3: Output of the element $I N$ generated by the processing (Eqs. 2 to 4$)$ of random inputs $(200 \mathrm{~Hz})$ from element $S S$ during $200 \mathrm{~ms}$. We assumed a rest potential of $-70 \mathrm{mV}$, and a synaptic delay of 0.5 ms. $E P S P=-68 \mathrm{mV}, I P S P=-72 \mathrm{mV}, w_{S E j}=0.9, t_{p}=5 \mathrm{~ms}, \tau_{P}=1.2 \mathrm{~ms}$, $\tau_{H}=1.3 \mathrm{~ms}$ (the other parameter values are given in Table 1 ).

Figs. $4 \mathrm{a}$ and $4 \mathrm{~b}$ illustrate the registered synaptic activities when $M N$ is simultaneously stimulated by all the other circuit elements during $200 \mathrm{~ms}$. In these figures, we see the depolarizing (Fig. 4a) and hyperpolarizing (Fig. 4b) effects coming from the application of equations 2 and 3 , respectively.

The changing character of the excitatory effects on the $M N$ element can be seen in Fig. 4a. In this figure, each pulse represents the temporal and spatial summation of several EPSPs generated from random $S$ and $S S$ outputs. The used $w_{S E j}$ value for $M N$ was 0.9 . In figure $4 \mathrm{a}$, it can also be seen that the sum of effects (pulse amplitudes) may reach large values, with a maximum of $46.59 \mathrm{mV}$. This is equivalent to the rising of the membrane potential from rest level (Table 1) to $-18.41 \mathrm{mV}$. According to Baker et al. [28], the amplitude of compound EPSPs evoked in a motoneuron by pyramidal tract stimulation can vary from 0.5 to $7.5 \mathrm{mV}$. Our value $(-18.41 \mathrm{mV})$ is down the low end of this range evoke EPSPs, but it is probable that the EPSP values are normally lower during spontaneous activity, since sensory-evoked responses usually present higher frequencies. Fig. $4 b$ shows a minimal temporal and spatial summation of the inhibitory synaptic effects of $-63.99 \mathrm{mV}$, 

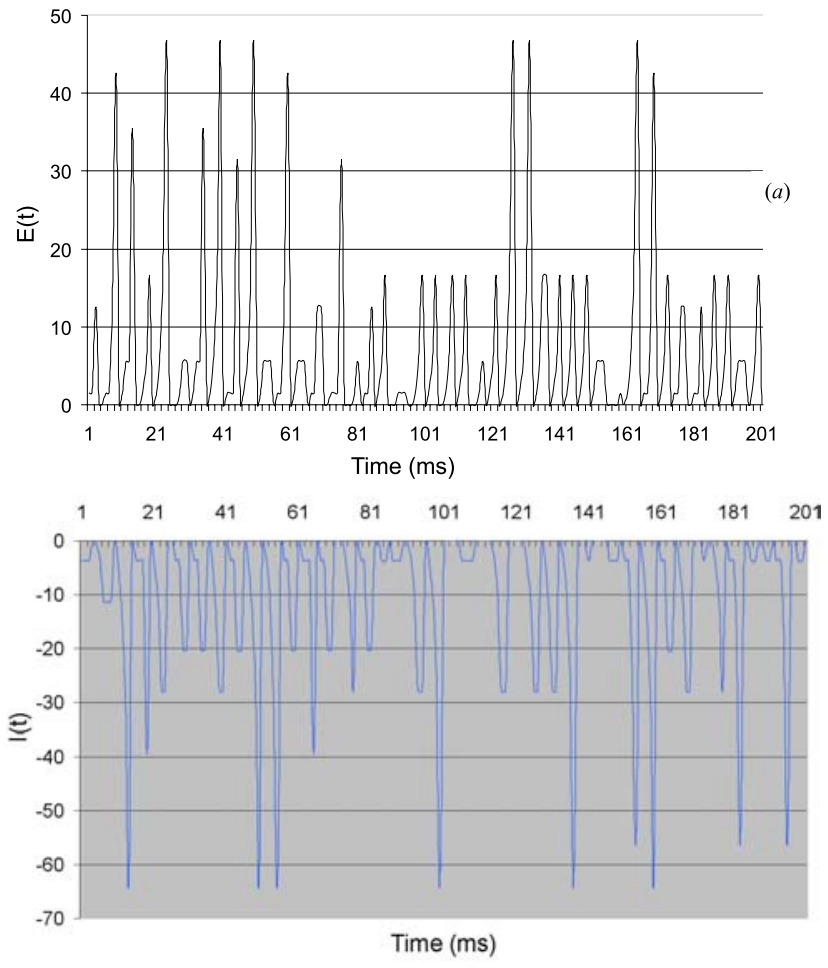

(b)

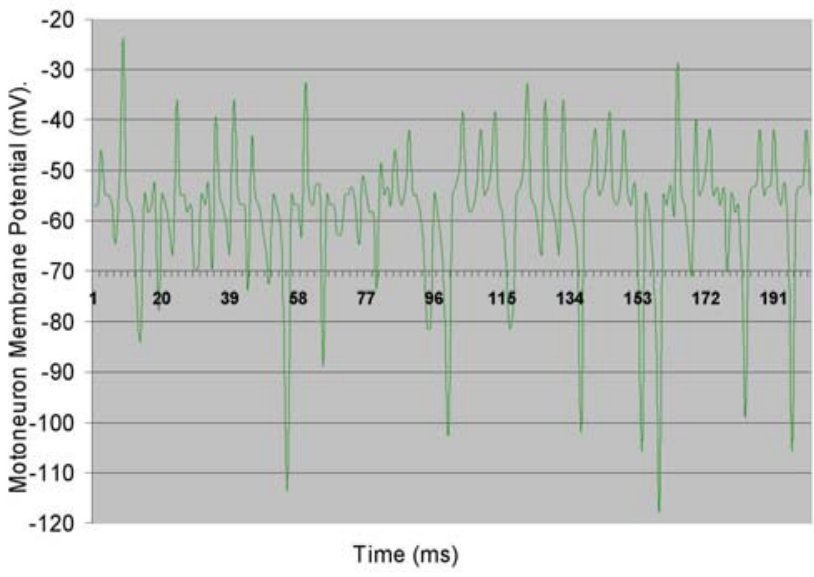

(c)

FIG. 4: Addition of (a) depolarizing and (b) hyperpolazing synaptic effects (Eqs. 2 and 3) on $M N$ for inputs coming from elements $S$, $I N$ and SS. (c) Estimated power spectrum of the $M N$ membrane potential resulting from simultaneously activities of the excitatory and inhibitory synapses during $200 \mathrm{~ms}$. We assumed a rest membrane potential of $-70 \mathrm{mV}$ (represented in the vertical axis by zero), and a synaptic delay of $0.5 \mathrm{~ms}$. $E P S P=-68 \mathrm{mV}, I P S P=-72 \mathrm{mV}, w_{S E j}=0.9$, $w_{S I j}=0.6$ (the other parameter values are given in Table 1).

which corresponds to a decrease in the membrane potential from rest to $-128.99 \mathrm{mV}$. We used $w_{S I j}=0.5$ for $M N$.

Figure $4 \mathrm{c}$ shows the time series of membrane potentials resulting from simultaneous activities of the excitatory and inhibitory synapses, during $200 \mathrm{~ms}$, on element $M N$. This graph represents the variation in membrane potential of element $M N$ due to algebraic summation of depolarizing and hyperpolar- izing PSPs. An irregular response in both the amplitude and frequency can be seen in this figure. The maximum value of the membrane potential estimated from Eqs. 4 (a) and (b) during $200 \mathrm{~ms}$ is $-36.8 \mathrm{mV}$; the minimum value is about -118.0 $\mathrm{mV}$.

An irregular $M N$ firing of mean frequency $120 \mathrm{~Hz}$ can be observed in Fig. 5. The threshold potential was $-40 \mathrm{mV}$ (see Table 1). The time interval to the first firing is about $2 \mathrm{~ms}$ after the arrival of the first $A P$ in the presynaptic membrane. The next two APs appear only after 8.0 and $24 \mathrm{~ms}$, respectively. These results are on the physiological scale. It is known that the maximum firing frequencies of $M N$ are usually smaller than $100 \mathrm{~Hz}$, but they may be up to $300 \mathrm{~Hz}$ at the beginning of an afferent stimulus [17,26,29-31].

Figures $6 \mathrm{a}, 6 \mathrm{~b}$ and $6 \mathrm{c}$ show the behavior of the average firing rate of $M N$ as a function of the time constant $\left(\tau_{E}\right)$, the excitatory $(E j)$ and inhibitory $(I j)$ postsynaptic potentials for a given value of the input rate $(100 \mathrm{~Hz})$, according to Eqs. 2 and 3. In each of these graphs, we observe a minimum value at which the element $M N$ begins firing. The frequency increases linearly until reaching a maximum value, and then it tends to remain constant or to decrease, which determines the value of the parameter related to the synaptic fatigue for that input frequency.

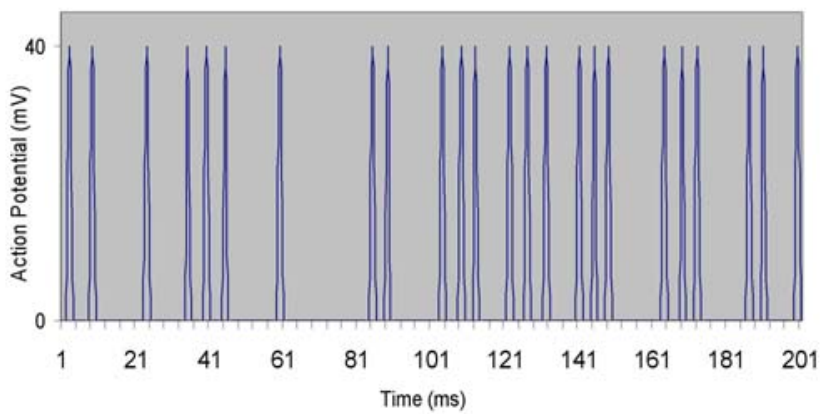

FIG. 5: $M N$ output derived from the sum of synaptic events for a random circuit stimulation $(200 \mathrm{~Hz})$ during $200 \mathrm{~ms}$. MN receives simultaneous inputs from $S, S S$ and $I N$. We assumed a rest membrane potential of $-70 \mathrm{mV}$, and a synaptic delay of $0.5 \mathrm{~ms}$. EPSP=-68mV, $I P S P=-72 \mathrm{mV}, w_{S E} j=0.9, w_{S I j}=0.6, t_{p}=5 \mathrm{~ms}, \tau_{P}=1.2 \mathrm{~ms}, \tau_{H}=1.3 \mathrm{~ms}$ (the other parameter values are given in Table 1). The first $M N$ firing occurs between $1.0 \mathrm{~ms}$ and $2.0 \mathrm{~ms}$, and the second firing takes place after $9.0 \mathrm{~ms}$. The mean frequency of synaptic events is $200 \mathrm{~Hz}$.

It is clear that the model equations are reductionist and that they are not reliable to describe more specific situations in the complex behavior of the nervous cells. In the literature, we find different kinds of approaches. One of these approaches is based on the ionic conductance properties; another approach is based on the quantitative wave properties of frequency responses from neurons interacting within the nervous system. Some authors consider these two approaches together [26,34]. We do claim that our approach leads to model equations which provide a very reasonable representation of the synaptic phe- 


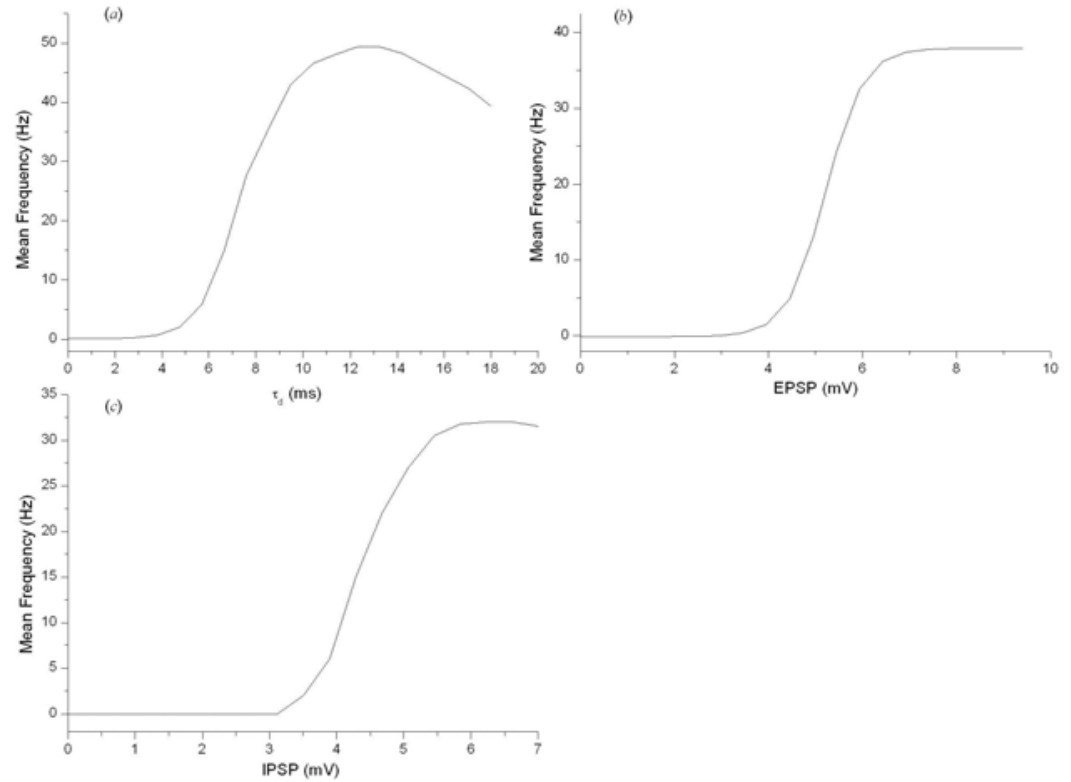

FIG. 6: Behavior of the average firing rate of $\mathrm{MN}$ as a function of $(a)$ the time constant $\left(\tau_{E}\right),(b)$ excitatory $(E j)$, and $(c)$ inhibitory $(I j)$ postsynaptic potentials, according to Eqs. 2 and 3 (the input rate is $100 \mathrm{~Hz}, w_{S E j}=0.9, w_{S I j}=0.6, \tau_{E}=10 \mathrm{~ms}$; the other parameter values are given in Table 1).

nomena.

In summary, our spinal reflex model produced results on a physiological scale. The model equations were able to describe the behavior of each circuit component. They were based on the synaptic transmission characteristics, including the typical parameters. The simulation environment can be easily expanded to include another neuronal geometry and additional membrane channels, as well as new cell and fiber populations. More realistic numerical values could have been used in the model equations if we were able to obtain more detailed data for the peripheral receptors, motoneurons and interneurons. We were unable to find experimental references for some of the parameters.

The data obtained in this work are being used in an ongoing study about backpropagation to a neural network based on a control modeling of spinal reflex patterns. In addition, the model will be expanded in order to include an explicit representation of the cortical neurons and other spinal afferences. Also, we are proposing a model a coupled oscillators to simulate the dynamical behavior of the sensoriomotor cortex and other structures within the suprasegmental nervous system.

This work was supported by Fundação de Amparo à Pesquisa do Estado do Rio de Janeiro, FAPERJ.
[1] M. G. Velarde, V. I. Nekorkin, V. B. Kazantsev, V. I. Makarenko, and R. Llinás, Neural Networks 15, 5 (2002).

[2] P. J. G. Lisboa, Neural Networks 15, 11 (2002).

[3] J. Peng, H. Qiao, and Z. Xu, Neural Networks 15, 95 (2002).

[4] C. Robert, C. Guilpin, and A. Limoge, J. Neurosci. Methods 79, 187 (1998).

[5] S. Rossignol, and R. Dubuc, Curr. Opin. Neurobiol. 4, 894 (1994)

[6] N. Donaldson, D. Rushton, and T. Tromans, Lancet 350, 711 (1997).

[7] R. E. Bekka, S. Boudaoud, and D. Chikouche, J. Neurosc. Meth. 116, 89 (2002).

[8] S. D. Prentice, A. E. Patla, and D. A. Stacey, J. Electromyogr. Kinesiol. 11, 19 (2001).

[9] S. D. Prentice, A. E. Patla, and D. A. Stacey, Exp Brain Res.
123, 474 (1998).

[10] C. Cortez and P. M. Bisch, Braz. J. Phys. 26, 604 (1996).

[11] K. M. Soares and C. Cortez, J. Theor. Biol. 169, 169 (1999).

[12] F. A. O. Cruz, F. S. D. S. Vilhena, and C. Cortez, Braz. J. Phys. 30, 403 (2000).

[13] E. Bizzi, F. A. Mussa-Ivaldi, and S. Giszter, Science 253, 287 (1991).

[14] A. Lansner and Ö. Ekeberg, Curr. Opin. Neurobiol. 4, 903 (1994).

[15] R. T. Lauer, P. H. Peckham, and K. L. Kilgore, Neuroreport. 10, 1767 (1999).

[16] A. C. Guyton and J. E. Hall, Human physiology and mechanisms of disease. $6^{\text {th }}$ edition. W. B. Saunders Co. Philadelphia, USA (1997).

[17] L. R. Johnson, Essential Medical Physiology. $2^{\text {th }}$ edition. 
Lippincott-Raven Publishers, Inc., Philadephia, USA (1998)

[18] L. M. Mendell and E. Henneman, J. Neurophysiol. 34, 171 (1971).

[19] W. A. Friedman, G. W. Sypert, J. B. Munson, and J. W. Fleshman, J. Neurophysiol. 46, 1349 (1981).

[20] R. W. Ryall, M. F. Piercey, and C. Polosa, J. Neurophysiol. 34, 700 (1971)

[21] R. E. Burke, B. Walmsley, and J. A. Hodgson, Brain Res. 160, 347 (1979)

[22] T. Chang, S. J. Schiff, T. Sauer, J. P. Gossard, and R.E. Burke, Biophys. J. 67, 671 (1994).

[23] R. B. Stein and M. N. Oguztoreli, Neuroscience 11, 231 (1984).

[24] R. J. Nelson, Curr. Opin. Neurobiol. 6, 801 (1996).

[25] V. S. Fénelon, B. Casasnovas, J. Simmers, and P. Meyrand, Curr. Opin. Neurol. 8, 705 (1998).

[26] D. P. Bashor, Biol. Cybern. 78, 147 (1998).

[27] C. L. Cleland, and W. Z. Rymer, J. Neurophysiol. 69, 1181 (1993).
[28] S. N. Baker, E. M. Pinches, and R. N. Lemon, J. Neurophysiol. 89, 1941 (2003).

[29] R. K. D. B. Powers and M. D. Binder, J. Neurophysiol. 75, 367 (1996).

[30] A. J. McComas, M. Mirsky, F. Velho, and A. Struppler, J. Neurol. Neurosci. Psychiatry, 42, 1091 (1979).

[31] W. R. Schlue, D. W. Richter, K-H. Mauritz, and A.C. Nacimiento, J. Neurophysiol. 37, 303 (1974).

[32] M. Andreasen, J.D.C. Lambert, J. Physiol. 507, 441 (1998)

[33] O. Sacchi, M. L. Rossi, R. Canella, and R. Fesce, J. Neurophysiol. 79, 727 (1998).

[34] P. Kudela, P. J. Franaszczuk, and G. K. Bergey, Biol. Cybern. 77, 71 (1997).

[35] E. F. Barrett, J. N. Barrett, and W. E. Crill, J. Physiol. 304, 251 (1980).

[36] J. S. Carp, J. Neurophysiol. 68, 1121 (1992). 